\title{
Informationen fur die Klinik
}

\section{Medizintechnîk}

C $\theta 2$-Laser für Chirurgie und Mikrochirurgie

Ein komplettes medizinisches C02-Laser-system «aus einer Hand» stellt Carl Zeiss, Oberkochen, unter dem Namen OPMILAS COzL vor.

Das System bietet die Möglichkeit, bei einer von 2-20 W variablen Leistung Eingriffe

durchzuführen, die bisher Systeme höherer Leistung erforderten. Bei kleinsten Spotgrö-ßen erreicht das Zeiss Lasersystem OPMILAS C02L Leistungsdichten von bis zu 450 $000 \mathrm{~W}$ pro Quadratzentimeter. Dies bietet den Vorteil schmaler Schnittkanäle, ge-rínger thermischer Belastung des umgeben-den Gewebes, geringer Karbonisation und präziser Entfernung kleinster Gewebevolu-mina.

Weitere Informationen erhalten Sie bei Carl Zeiss, Postfach 1369/1380, D-7082 Ober$\mathrm{r}$

Kongreßbericht

«Highlights auf Audiokassette»

Der 23. Jahreskongreß der Amerikanischen Gesellschaft für Klinische Onkologie, der in Atlanta stattfand, bot ein breitgefächertes Themenspektrum. Dazu zählten u.a. ein Überblick über aktuelle therapeutische Möglichkeiten bei Krebserkrankungen im Kindesalter, eine kritische Würdigung des therapeutischen Potentials von Biological Response Modifiers sowie chemotherapeu-tische Behandlungsstrategien bei Leukämien und malignen Non-Hodgkin-Lymphomen, beim metastasierten Mamma-karzinom und beim fortgeschrittenen kolo-rektalen Karzinom.

Eine nunmehr vorliegende Audiokassette faßt diese Themen zusammen. Zusätzlich enthält sie eine Diskussion mit namhaften bundesdeutschen Kongreßteilnehmern, die die Ergebnisse aus íhrer Sicht kommentie-ren.

Die Kassette kann kostenlos bei Cyanamid GmbH, Lederle Arzneimittel, Herrn Dr. Enghofer, Pfaffenrieder Str. 7, D-8190 Wolfratshausen, abgerufen werden.

Diagnostika

Neuer Katalog

Auf über 150 Seiten präsentiert Sigma Diagnostics das komplette Angebot an Reagen-zienSätzen für das klinisch-medizinische Laboratorium.

Alle Produkte werden methodisch beschrie-ben, enthalten Angaben über Testzusammensetzungen sowie erforderliche Zusatz-reagenzien und sind mit Bestellbezeichnun-gen und Preisangaben versehen. Den neuen Katalog erhalten Sie bei: Sigma Diagnostics, Grünwalder Weg 30, D-8024 Deisenhofen, Tel. (089) 61301-0, Telex 528252.

Preisverleihungen

Preisträger des Artur-Pappenheim-Preises 1987

Insgesamt wurden 5 Arbeiten eingereicht. Hieraus wurde die Arbeit von Herrn F. Herrmann/Mainz über «Myelopeotisch relevante Zytokine: Zelluläre Herkunft, Induktionserfordernisse, Interaktionsmecha-nismen und biologische Aktivität» vom Gut- 
achtergremium zur Auszeichnung ausge-wählt. Herrn Herrmann wurden Urkunde und ArturPappenheim-Preis in der Eröff-nungssitzung am 4.10.1987 überreicht.

Preisträger des Vincenz-Czerny-Preises 1987

Aus insgesamt 7 Bewerbungen ging die Arbeit der Herren E.D.Kreuser und W. D. Hetzel/Ulm über «lnzidenz und Pro-gnose reproduktiver und endokriner Funk-tionsstörungen nach kurativer zytostatischer Chemotherapie bei Patienten mit malignen Erkrankungen» als die beste im Sinne der Ausschreibung hervor. Beiden Herren wurden am 4.10.1987 Urkunde und Preis überreicht. Medizin-Nobelpreis 1987

Wie das schwedische Nobelkommitee am 12. Oktober 1987 mitteilte, wird dieses Jahr Prof. Dr. Susumu Tonegawa am Massachusetts Institute of Technology (MIT) mit dem Nobelpreis für Physiologie und Medizin aus-gezeichnet.

Prof. Tonegawa, geboren am 5. September 1939 in Nagoya, Japan, studierte zunächst in Kyoto und promovierte 1968 an der Univer-sität von Kalifornien in San Diego. Am 1. Februar 1971 kam er an das Basler Insti-tut für Immunologie zu Prof. Niels K. Jerne. Ende September 1981 ging Tonegawa als. Professor für Biologie von Basel an das Krebsforschungszentrum des MIT, wo er auch heute arbeitet.

Angeregt von Prof. Jernes Ideen, klärte Dr. Tonegawa in Basel in den Jahren 1975 bis 1981 mit bahnbrechenden Experimenten als erster die Struktur und Organisation der Antikörper-Gene auf. Damit konnte erklärt werden, wie es den B-Zellen des Immunsy-stems möglich ist, mit nur einigen hundert Genen Milliarden verschiedener Antikörper zu konstruieren und damit auf eine schier endlose Zahl von Antigenen die «passende» Immunantwort zu finden. Dr. Tonegawa und seine Kollegen im Basler Institut für Immunologie wiesen ab 1975 nach, daß diese AntikörperGene in der Keimbahn nicht aus einer zusammenhän-genden Information, das heißt einem Stuck DNS, bestehen, sondern daß sie sich aus 3 oder 4 Informationsteilen zusammensetzen. Wenn ein B-Lymphozyt sich entschließt, Antikörper zu produzieren, werden diese Informationsteile jeweíls neu geordnet und miteinander verbunden. Tonegawas Studien über die Struktur der 4 einzelnen Informationsteile ergaben, daß jedes dieser Teile selber wieder aus einer Gruppe von nahe benachbarten Informationsteilen besteht. Daraus ergeben sich sehr viele Kombinationsmöglichkeiten für die Gene, welche die Struktur von Antikörpern bestimmen. Die große Zahl von Antikörper-Strukturen, die somit durch Genrekombination gebildet werden, erhöht sich noch um sogenannte somatische Mutationen. Hierbei handelt es sich um spontane Veränderungen, die in der DNS der antikörperbildenden Zellen statt-finden; diese somatischen Mutationen werden nicht Teil der von Generation zu Generation weitergegebenen genetischen Information.

Verantwortlich für die Zusammenstellung: Frans H. M. Stroeks, Puchheim b.München 